Meta

Journal des traducteurs

Translators' Journal

\title{
Description prédictionnairique de trois mots du français québécois : francophone, anglophone et allophone
}

\section{Pierre Martel}

Volume 39, numéro 4, décembre 1994

Hommage à Bernard Quemada : termes et textes

URI : https://id.erudit.org/iderudit/003586ar

DOI : https://doi.org/10.7202/003586ar

Aller au sommaire du numéro

Éditeur(s)

Les Presses de l'Université de Montréal

ISSN

0026-0452 (imprimé)

1492-1421 (numérique)

Découvrir la revue

Citer cet article

Martel, P. (1994). Description prédictionnairique de trois mots du français québécois : francophone, anglophone et allophone. Meta, 39(4), 615-624.

https://doi.org/10.7202/003586ar
Résumé de l'article

Monsieur Bernard Quemada, dans un article intitulé « La nouvelle lexicographie », suggérait une phase " de rédaction prédictionnairique » à celle de la rédaction d'un article de dictionnaire. À l'Université de Sherbrooke, nous avons mis en œuvre une telle description prédictionnairique. La BANQUE DE DONNÉES TEXTUELLES DE SHERBROOKE contient plus de six millions de mots et elle est composée de divers textes : oraux, littéraires et non littéraires. Afin de décrire les unités lexicales contenues dans cette banque de données, nous avons mis au point une fiche prédictionnairique pour les substantif $s$ fréquents qui s'y trouvent. Je me propose de situer notre travail prédictionnairique et de l'illustrer au moyen de quelques termes fréquents de notre banque et caractéristiques de certains textes québécois. Je choisirai ceux relatifs à l'expression d'une langue : francophone (par opposition à français ), anglophone (par opposition à anglais ) et allophone . 


\title{
DESCRIPTION PRÉDICTIONNAIRIQUE DE TROIS MOTS DU FRANCCAIS QUÉBÉCOIS : FRANCOPHONE, ANGLOPHONE ET ALLOPHONE'
}

PIERRE MARTEL

Université de Sherbrooke, Sherbrooke, Canada

\begin{abstract}
Résumé
Monsieur Bernard Quemada, dans un article intitulé "La nouvelle lexicographie», suggérait une phase «de rédaction prédictionnairique» à celle de la rédaction d'un article de dictionnaire.

À l'Université de Sherbrooke, nous avons mis en cuvre une telle description prédictionnairique. La BANQUE DE DONNÉES TEXTUELLES DE SHERBROOKE contient plus de six millions de mots et elle est composée de divers textes : oraux, littéraires et non littéraires. Afin de décrire les unités lexicales contenues dans cette banque de données, nous avons mis au point une fiche prédictionnairique pour les substantifs fréquents qui s'y trouvent.

Je me propose de situer notre travail prédictionnairique et de l'illustrer au moyen de quelques termes fréquents de notre banque et caractéristiques de certains textes québécois. Je choisirai ceux relatifs à l'expression d'une langue : francophone (par opposition à français), anglophone (par opposition a anglais) et allophone.
\end{abstract}

\begin{abstract}
In an article entitled "La nouvelle lexicographie", Bernard Quemada suggests that a phase of "pre-dictionary writing" precede the phase of dictionary-entry writing.

At the Universite de Sherbrooke, we have set up such a pre-dictionary description project. The BANQUE DE DONNEEES TEXTUELLES DE SHERBROOKE contains more than five million words and is composed of a variety of oral, literary, and non-literary texts. In order to describe the lexical units contained in this data bank, we have developed a predictionary data sheet for frequently occurring nouns.

I propose to describe our pre-dictionary work and to illustrate it by means of three frequently occurring terms in our bank that are characteristic of certain Quebec texts: francophone, anglophone, and allophone.
\end{abstract}

Les mots se terminant en -phone et désignant ceux «qui parlent habituellement la langue $\mathrm{X}$ » comme francophone, germanophone, lusophone, hispanophone, anglophone, etc. forment une série d'unités lexicales spécialisées. Ils sont peu nombreux à figurer dans les dictionnaires courants et, quand ils s'y trouvent, ils sont en général monosémiques.

Prenons, par exemple, le mot francophone, le plus fréquent de tous et pour cause. Selon le Dictionnaire historique de la langue française (Éditions Robert), il est récent: il daterait de 1930. Dans la $1^{\text {re }}$ édition du Grand Robert (1960), francophone est consigné sous l'entrée «Franco- Préfixe...» et n'est défini que par les quelques mots suivants: «adj. et $\mathrm{n}$. Dont le français est la langue usuelle». Même s'il est devenu moins rare aujourd'hui en France, son emploi semble demeurer relativement restreint.

En français québécois, par contre, outre francophone, deux autres mots de cette série, anglophone et allophone, sont particulièrement fréquents et importants car ils sont 
omniprésents dans les études «démolinguistiques» effectuées par les organismes de la Charte de la langue française. En effet, dans la transmission des informations linguistiques, notamment dans les médias écrits, l'utilisation de ces mots est constante. Dans la Banque de données textuelles de Sherbrooke (BDTS) (Martel et Théoret. 1991 : 123, 143 et voir ci-dessous), les mots francophone et anglophone ont une fréquence moyenne: francophone compte 331 occurrences et anglophone, 234 sur un total de cinq millions de mots; le mot allophone ne compte que 13 occurrences.

Ces mots se retrouvent principalement dans des textes journalistiques et sociopolitiques. La plupart de leurs occurrences relèvent de textes de niveau soutenu (articles de journaux, éditoriaux, articles scientifiques, mémoires, etc.) qui représentent, à mon avis, des textes tirés de la langue standard au Québec. En examinant les emplois de ces vocables, nous relevons des sens inconnus en France, par exemple, un «hôpital francophone». Ce syntagme n'est pas sans me rappeler l'étonnement d'un Français à la suite d'une demande d'un Québécois, dans une boulangerie en France, qui voulait acheter un «pain français»!

Ces mots, francophone, anglophone et allophone, nous ont donc paru intéressants à analyser et à décrire selon une méthode récemment adoptée à Sherbrooke que nous appelons «description ou analyse prédictionnairique» du vocabulaire du français québécois. Cette méthode appliquée plus particulièrement aux unités lexicales largement attestées dans la BDTS, a été mise au point à Sherbrooke il y a quelques années comme suite à un énoncé de Bernard Quemada:

[...] l'analyse pré-dictionnairique des données, c'est-à-dire la description (ou la mise en évidence) de leurs caractéristiques. Elle représente le travail proprement lexicographique au cours duquel l'unité-mot est relevée, identifiée et examinée dans ses divers contextes linguistiques et extralinguistiques, sans choix ni orientations préalables. Les contours sémantiques sont délimités sans faire intervenir de préoccupations de type homonymique/polysémique. Les conditions d'usage sont en revanche illustrées avec le plus de précision possible (lexicalisation, distributions et relations fonctionnelles, valeurs d'emploi, etc.).» (Quemada $1990: 64)$

Nous avons fait nôtre la proposition de Bernard Quemada en l'adaptant à notre besoin, en particulier en ce qui concerne les définitions et la polysémie. La publication de l'Hommage à Bernard Quemada me donne l'occasion de présenter notre analyse en l'illustrant des mots francophone, anglophone et allophone.

\section{L'ANALYSE PRÉDICTIONNAIRIQUE}

L'Université de Sherbrooke dispose d'un Centre d'analyse et traitement informatisé du français québécois, appelé CATIFQ, regroupant six professeurs. Dans le cadre de leurs travaux, ils ont constitué une Banque de données textuelles. Cette dernière comprend à l'heure actuelle plus de 3000 textes différents, postérieurs à 1960, et au-delà de 5 millions de mots. Ces textes représentent un échantillon que nous voulons représentatif de la langue parlée et écrite du Québec. Nous avons donc des textes de langue orale et des textes de langue écrite; des textes littéraires et non littéraires (pour la description de la typologie des textes, voir Martel 1993). Un des objectifs poursuivis est de procéder à la description prédictionnairique des unités lexicales contenues dans la BDTS et de fournir ainsi aux futurs lexicographes québécois toutes les informations dont ils auront besoin pour rédiger n'importe quel type de dictionnaires. Aussi, l'analyse prédictionnairique vise-t-elle à présenter l'ensemble du matériel contenu dans la BDTS, en réunissant, pour chaque vocable, toutes les données lexicologiques pertinentes et en présentant ces dernières de manière systématique. 
Pour ce faire, nous avons établi une procédure rigoureuse et les données sont organisées dans un système informatisé qui permet une recherche multifonctionnelle. Nous avons aussi pris soin de définir les sens des mots en précisant le pont sémantique entre les acceptions, pont qui constitue la base de la structure de l'article; toutes les valeurs grammaticales et pragmatiques sont notées. Ce travail nécessite l'examen de toutes les occurrences du mot contenues dans la BDTS pour en distinguer les emplois. Ces informations sont complétées par la consultation des ouvrages de référence susceptibles d'ajouter, de confirmer ou de préciser les sens du mot ou d'y ajouter diverses informations métalinguistiques.

Voici, très brièvement, un aperçu des éléments contenus dans la fiche. Elle comporte deux parties: la fiche vocable et la fiche acception (Voir Annexe).

La fiche vocable comprend les éléments fixés : le vocable (lemme), la catégorie grammaticale, la prononciation, la graphie, la flexion, le composé (s'il y a lieu), l'étymologie, les homographes, les homophones, les références bibliographiques.

La fiche acception regroupe les éléments suivants: la classe (d'objet pour le nom), la définition, les exemples, les synonymes, les antonymes, les coocurrences ${ }^{2}$, les compléments (les éléments dépendants figurant à la droite du mot), la structure (les éléments grammaticaux se trouvant à gauche ou à droite), la distribution (position antérieure/postérieure $\mathrm{du}$ vocable), les étiquettes normatives, les marques d'usage et enfin une rubrique appelée «notes» pour toute information supplémentaire. Quelques autres éléments relatifs au référent sont parfois ajoutés. Les expressions font l'objet d'une fiche indépendante, liée au sens simple correspondant (Martel et Théoret 1993).

Je précise que les acceptions décrites sur nos fiches ne correspondent pas nécessairement, dans notre esprit, aux sens du mot tels qu'ils seraient rédigés dans un futur dictionnaire. Nous apportons en effet des distinctions sémantiques selon des règles précises, lesquelles sont essentielles à cette phase de description lexicographique. L'auteur d'un futur dictionnaire, bénéficiant de notre analyse sémantique, préférera peut-être reformuler certaines définitions, en regrouper certaines, en ignorer d'autres, etc. compte tenu du type de dictionnaire qu'il rédigera et du public auquel il le destinera.

\section{LES MOTS FRANCOPHONE, ANGLOPHONE ET ALLOPHONE EN FRANÇAIS QUÉBÉCOIS}

\subsection{Francophone}

Dans la dernière édition (1993) du Nouveau Petit Robert (PR) ${ }^{3}$, l'article francophone est présenté avec deux sens seulement:

1. Qui parle habituellement le français au moins dans certaines circonstances de la communication comme langue première ou seconde. Les Africains francophones. (En parlant d'un groupe, d'une région) Dans lequel le français est pratiqué en tant que langue maternelle, officielle ou véhiculaire (même si les individus ne parlent pas tous le français). La communauté francophone. La partie francophone de Montréal. La Suisse francophone. $\rightarrow$ romand. Université francophone d'Alexandrie. N. (surtout au plur.) Personne appartenant à une telle communauté. Les francophones de Belgique $(\rightarrow$ wallon), de Louisiane $(\rightarrow$ cajun), du Canada $(\longrightarrow$ acadien, québécois $)$. 2. Relatif à la francophonie. La littérature francophone.

L'analyse des divers contextes d'utilisation préalable à la rédaction de la fiche prédictionnairique a révélé d'autres sens de ce mot en français québécois. Compte tenu du peu d'espace dont je dispose, je me limiterai ici à présenter les définitions du mot, qui représentent l'élément principal de la fiche, les exemples et les cooccurrences d'utilisation du mot. 
FRANCOPHONE n. m. et f., adj.

Fiches acception :

Déf. : n. $\bullet$ 1. Personne dont la langue maternelle ou la langue d'usage est le français.

Ex.: "Une façon de faciliter l'insertion harmonieuse de ces nouveaux citoyens dans une réalité marquée par la fragilité démographique des francophones.» (Pierre Gravel, La Presse, édit., 3-10-90)

Déf.: adj. - 2.a. Dont la langue maternelle ou la langue d'usage est le français (appliqué aux personnes).

Ex.: "Ce choix, tout récent pour de nombreux Québécois francophones, est l' aboutissement d'un processus historique.» (Alain Dubuc, La Presse, édit., 5-05-91) «Plusieurs écoles font des efforts louables pour remplir le vide: clubs de lecture, écoute de chansonniers francophones, etc.» (Agnès Gruda, La Presse, édit., 9-05-91)

Cooc.: Africain, cadre, Canadien, chansonnier, citoyen, clientèle, fédéraliste, fonctionnaire, garagiste, habitant, immigrant, joueur, journaliste, Libanais, locuteur, ministre, oreille, parlementaire, personnalité, producteur, Québécois, vedette, vice-président.

Déf.: - 2.b. Qui a pour facteur de regroupement le fait que ceux qui en font partie ont pour langue maternelle ou langue d'usage le français (appliqué à un groupe de personnes).

Ex.: "La moitié des cinquante pays ou territoires faisant partie de la communauté francophone se trouvent en Afrique.» (Pierre Gravel, La Presse, édit., 22-02-91)

Cooc.: base, bloc, camp, collectivité, communauté, diaspora, entité, groupe, majorité, minorité, population, société.

Déf. : * 3.a. Qui est typique à un groupe dont les membres ont pour langue maternelle ou langue d'usage le français.

Ex.: "La culture francophone sur ce continent n'a plus de sens si on se met au service d' une autre culture, américaine ou française.» (Réginald Martel, Livres 22-12-90)

Cooc. : culture, identité, nature, opinion publique, origine, réalité, souche.

Déf. : 3.b. Qui dépend de, qui est relatif à des personnes ayant pour langue maternelle ou langue d'usage le français.

Ex.: «Dans le secteur manufacturier par exemple, la part de propriété francophone est encore relativement faible.» (Mémoire présenté à la Commission Bélanger-Campeau, Q2 38) Cooc.: audience, contrôle, côté, courant, Félix, langue, marché, propriété, vent.

Déf.: - 4.a. Qui fonctionne en français et qui est administré par des personnes dont la langue maternelle ou la langue d'usage est le français.

Ex.: "Actuellement, les enfants de langue maternelle autre que le français sont majoritaires dans 28 des 178 écoles francophones de la CECM.» (Agnès Gruda, La Presse, édit., 14-05-91) Cooc. : club, école, entreprise, équipe, université.

Déf. : - 4.b. Qui est rédigé en français, qui se déroule en français.

Ex. : "C'est une histoire qui a passé inaperçue dans la presse francophone et qui a été complètement ignorée par les innombrables bilans de fin d'année.» (Francine Pelletier, La Presse, édit., 5-01-91)

Cooc. : chaîne, chanson, contenu, gala, journaux, média, presse, production, sommet, télévision, thêâtre.

Déf.: - 5.a. Dont la majorité des locuteurs ont pour langue maternelle ou langue d'usage le français ou dont la langue officielle est le français (en parlant d'une région géographique).

Ex.: «Une centaine d'artistes et de scientifiques d'Algérie, du Maroc, de France, d'Italie, de Belgique, du Canada, des États-Unis et, bien sûr, de plusieurs pays francophones d'Afrique.» (Francine Grimaldi, La Presse, Arts 16-12-90)

Cooc. : État, Montréal, pays, province, quartier, région, ville.

Déf.: - 5.b. Dont la majorité des locuteurs ont pour langue maternelle ou langue d'usage le français ou dont la langue officielle est le français (en parlant d'une zone située à l'intérieur de la région géographique concernée). 
Ex.: «Pour l'instant le documentaire réalisé par Mario Bonenfant sera aussi diffusé en Europe par TV5 et dans toute l'Afrique francophone via les télévisions nationales.» (Francine Grimaldi, La Presse, Arts 14-10-90)

Cooc. : Afrique, Canada, espace, monde, Québec.

Les auteurs du plus récent dictionnaire du français québécois, le Dictionnaire québécois d'aujourd' hui (DQA), définissent ainsi le mot francophone:

adj. et $n$. Qui parle le français, soit comme langue maternelle, officielle ou seconde. Les Africains francophones. - N. Les francophones de l'Ouest. Les francophones et les allophones. - Où le français est en usage. L'Afrique francophone. C'est un quartier francophone.

Les nombreuses coocurrences fournies par la BDTS ont permis de distinguer plus de 5 sens et sous-sens. Francophone en français du Québec s'emploie ainsi dans des contextes beaucoup plus variés que ne l'indiquent les deux dictionnaires PR et DQA.

\subsection{Anglophone}

Dans le Nouveau Petit Robert, on lit la définition suivante au mot anglophone:

adj. et n. Où l'on parle l'anglais. Pays anglophone. — Qui parle la langue anglaise. Canadien anglophone. $\mathrm{N}$. Un, une anglophone.

ANGLOPHONE n. m. et f., adj.

Fiches acception:

Déf.: n. 1. Personne dont la langue maternelle ou la langue d'usage est l'anglais.

Ex.: «Les anglophones ressentent à l'endroit du pouvoir politique québécois un ressentiment évident et se sentent souvent à l'extérieur de la vie québécoise.» (Alain Dubuc, La Presse, édit., 8-09-90)

Déf.: adj. • 2.a. Dont la langue maternelle ou la langue d'usage est l'anglais (appliqué aux personnes).

Ex.: «Entre les numéros, on a donc vu un genre de rétrospective des grands comiques anglophones qui ont participé au festival au cours des dernières années.» (D. Bonneau, La Presse, Consommateurs, 22-07-90)

Cooc. : adolescent, artiste, auteur, Canadien, chanteur, chroniqueur, comique, commentateur, consœur, créditiste, dirigeant, fonctionnaire, homme, homme d'affaires, joueur, juge, ministre, Nord-Américain.

Déf.: - 2.b. Qui a pour facteur de cohésion le fait que ceux qui en font partie ont pour langue maternelle ou langue d'usage l'anglais (appliqué à des groupes de personnes).

Ex.: «Montréal a subi la perte d'une partie de son élite économique anglophone ainsi qu' une perte importante de nouveaux immigrants.» (D. Senik, Technologie, 19)

Cooc.: bastion, bloc, communauté, élite, establishment, intelligentsia, majorité, minorité, population, société.

Déf.: - 3.a. Qui est typique à un groupe dont les membres ont pour langue maternelle ou langue d'usage l'anglais.

Ex.: "Son nouveau rôle l'oblige à des compromis, à des concessions, tant à l'intérieur qu'à l'extérieur de sa députation et de son parti qu'auprès de l'opinion publique aussi bien francophone qu'anglophone.» (Claude Masson, La Presse, édit., 16-02-91)

Cooc.: opinion publique.

Déf. : - 3.b. Qui dépend de, qui est relatif à des personnes ayant pour langue maternelle ou langue d'usage l'anglais.

Ex.: "L'environnement du travail y "est et sera toujours unilingue anglophone".» (Pierre Gravel, La Presse, édit, 27-03-91)

Cooc.: contrôle, environnement, milieu, pouvoir, propriété, solitude, unilinguisme. 
Déf.: 4.a. Qui fonctionne en anglais et qui est administré par des personnes dont la langue maternelle ou la langue d'usage est l'anglais.

Ex.: "Actuellement, le réseau anglophone compte quelque 1600 élèves qui n'ont pas encore reçu le feu vert à l' enseignement en anglais." (Agnès Gruda, La Presse, édit., 28-05-91)

Cooc. : cégep, école, institution, organisme, réseau scolaire, université.

Déf. : 4.b. Qui est rédigé en anglais, qui se déroule en anglais.

Ex. : «Les producteurs Jamie Brown et Robin Spry, forts de leur excellent produit, récidivent et se lancent dans la production pour la télévision anglophone.» (Francine Grimaldi, La Presse, Arts, 16-09-90)

Cooc.: média, radio, station, télévision.

Déf.: - 5.a. Dont la majorité des locuteurs ont pour langue maternelle ou langue d'usage l'anglais ou dont la langue officielle est l'anglais (en parlant d'une région géographique).

Ex.: "Le prix de vente des habitations de luxe, notamment les condominiums, accuse des baisses importantes, surtout dans les localités anglophones de l' lle de Montréal.» (F. Bernard, La Presse, Habitation, 23-03-91)

Cooc. : Amérique, continent, État, localité, pays, province.

Déf.: 5.b. Dont la majorité des locuteurs ont pour langue maternelle ou langue d'usage l'anglais ou dont la langue officielle est l'anglais (en parlant d'une zone située à l'intérieur de la région géographique concernée).

Ex.: "Une très grande majorité du Canada anglophone était farouchement opposée à l' accord du Lac Meech.» (Mémoire présenté à la Commission Bélanger-Campeau, Université, L 4).

Cooc. : Canada, monde.

Dans le DQA, anglophone reçoit la définition suivante :

adj. et n. Qui est de langue anglaise. La presse anglophone. - Un, une anglophone. - Où l'anglais est en usage. Les quartiers anglophones de Montréal.

Le mot anglophone est décrit de façon identique dans le PR et le DQA; il est réduit à un seul sens et l'article est beaucoup plus bref que celui de francophone. Selon les données de la BDTS, les deux mots, anglophone et francophone, comptent autant de sens et de sous-sens et les cooccurrences d'anglophone sont à peine moins nombreuses que celles de francophone.

\subsection{Allophone}

Dans le Nouveau Petit Robert, on lit la définition suivant :

n. et adj. [...] 1.Personne dont la langue matemelle est une langue étrangère, dans la communauté où elle se trouve. Adj. Des étudiants allophones. 2. N.m. Se dit de formes phonétiquement différentes...

\section{ALLOPHONE n. m. et f., adj.}

Fiches acception:

Déf.: n. $\bullet$ 1. Personne dont la langue maternelle ou la langue d'usage est autre que la (les) langue(s) officielle(s) ou autochtone(s) de la communauté où elle vit.

Ex.: «Cette décision met aussi en lumière l'importance pour la majorité au Québec de persuader les allophones, qui s'établissent ici en nombre croissant, de choisir le français, plutôt que l'anglais, comme principale langue d'usage.» (Pierre Gravel, La Presse, édit., 31-08-90)

Déf. : adj. $\bullet$ 2.a. En parlant d'une personne : dont la langue maternelle ou la langue d'usage est autre que la (les) langue(s) officielle(s) ou autochtone(s) de la communauté où elle vit. Ex.: "Cette transformation est plus visible dans nos écoles, surtout montréalaises, où près de $50 \%$ des élèves sont allophones et proviennent de 88 pays différents." (Mémoire présenté à la Commission Bélanger-Campeau, Q1 6)

Cooc. : élève, Québécois. 
Déf. : - 2.b. En parlant d'un groupe de personnes : qui est caractérisé par le fait que ceux qui en font partie ont pour langue maternelle ou d'usage une langue autre que la (les) langue(s) officielle(s) ou autochtone(s) de la communauté où ils vivent.

Ex.: «Respecter les régionalismes et protéger les minorités éparses à travers le Canada, soient-elles autochtone, française, anglaise ou allophone.» (Mémoire présenté à la Commission Bélanger-Campeau, Émile Colas, 5)

Cooc.: minorité.

Déf. : * 3. Qui dépend de, qui est relatif à des personnes dont la langue maternelle ou la langue d'usage est autre que la (les) langue(s) officielle(s) ou autochtone(s) de la communauté où elles vivent.

Ex.: «Comment parviendrons-nous à établir un système de garderies accordé aux particularités de notre mentalité, de notre géographie, de notre vie sociale et économique alors que nous sommes si distincts de l'autre solitude anglophone ou allophone?» (Mémoire présenté à la Commission Bélanger-Campeau, Club souver., 3)

Cooc.: solitude.

Déf. : 4. Qui fonctionne dans une langue autre que la (les) langue(s) officielle(s) ou autochtone(s) de la communauté et qui est administré par des gens dont la langue maternelle ou la langue d'usage est autre que la (les) langue(s) officielle(s) ou autochtone(s) de la communauté où ils vivent.

Ex.: "Dans un tel contexte, on comprend que les "organismes" anglophones ou allophones refusent de voir dans les membres de la commission d"origine autre que francophone, "leurs représentants"." (Pierre Vennat, La Presse, édit., 17-09-90)

Cooc. : organisme.

Le mot allophone constitue une entrée séparée dans le DQA et l'article se lit ainsi :

n. et adj. Personne qui a pour langue maternelle une langue autre que celle(s) officiellement en usage ou autochtone(s) dans le pays où elle vit. Les allophones de Montréal. - Adj. Relatif aux allophones. Un quartier allophone. Une ministre allophone. $\rightarrow$ anglophone, arabophone, francophone.

Ce mot est moins fréquent en français québécois que les deux précédents et l'étendue des cooccurrences dans la BDTS moins grande; aussi les sens et sous-sens sont-ils moins nombreux. Par ailleurs, il faut remarquer que le PR définit allophone sans faire mention des langues autochtones. À l'instar des auteurs du DQA, j'ai exclu dans la définition du mot les langues amérindiennes («autre que la langue officielle ou autochtone»), comme le précise la situation socio-politique du Québec.

\section{CONCLUSION}

Comme on le voit, les acceptions tirées des citations de la BDTS sont beaucoup plus nombreuses et riches que les sens décrits dans le Nouveau Petit Robert. De plus, on ne peut que s'étonner du nombre élevé de cooccurrences avec lesquelles ces trois mots se retrouvent. Comme suite à notre analyse, il apparaît clairement que leurs emplois en français québécois sont beaucoup plus variés qu'en français de France. La description du DQA, sur ce point, est aussi incomplète (je rappelle que l'absence de banques de données textuelles explique les lacunes de ce dictionnaire).

Dans cet article, je n'ai présenté la description que de trois mots du français québécois tirés de textes reflétant le français québécois standard ou français standard d'icit ${ }^{4}$. Ils ne représentent certes pas encore un échantillon suffisant pour attester l'existence de ce niveau spécifique. Mais notre travail sur des centaines d'autres unités, analogues à celles-ci, se poursuit, et la description prédictionnairique montrera ainsi que le français québécois ne se distingue pas uniquement du français de France par des mots et emplois de niveaux 
«familier» et «populaire», mais également par ceux du niveau «standard», comme l'affirmait le Conseil de la langue française en 1990:

En effet, il s'est progressivement établi au cours des dernières années au Québec -- à peu près depuis le début de la Révolution tranquille - un usage public du lexique, aussi bien dans la langue êcrite que dans la langue parlée [...]. Ce modèle, pourtant incomplet et imprécis, conduit déjà à distinguer des registres de discours et à y inclure les mots selon la valeur sociale ou stylistique qu'on leur accorde. (Conseil de la langue française 1990: 29)

De plus, les exemples décrits dans cet article montrent déjà l'intérêt, voire la nécessité, d'une banque de textes québécois pour une description du français québécois. Sans un vaste corpus de textes représentatifs de la langue d'ici, toute description lexicographique sera nettement incomplète et surtout, largement insatisfaisante aux Québécois et Québécoises. Je ne peux que rappeler encore ici la principale recommandation formulée par le Conseil de la langue française en ce sens:

Le Conseil de la langue française propose de rassembler en un seul lieu un immense corpus informatisé de textes, écrits et oraux, du français du Québec, qui servirait de matériau de base à la fabrication de dictionnaires du français québécois, d'ouvrages langagiers pour les établissements d'enseignement, de source documentaire pour les littéraires, les historiens, les linguistes, de banque de citation pour les utilisateurs et les enseignants de la langue. (Conseil de la langue française 1990:35)

La Banque de données textuelles de Sherbrooke et la description prédictionnairique que nous effectuons des vocables qu'elle contient s'inscrivent dans cette voie et permettent d'ores et déjà de montrer, exemples à l'appui, la nécessité d'une telle banque.

L'analyse prédictionnairique d'unités lexicales poursuivies à Sherbrooke constitue une étape essentielle, selon nous, de la description lexicographique du français québécois. Cette phase prédictionnairique, suggérée par Bernard Quemada, est déjà bien lancée au CATIFQ. Nous disposons en ce moment de quelques milliers de fiches décrivant plusieurs centaines de vocables. Notre objectif est de décrire le vocabulaire fréquent et complexe du français québécois d'ici quelques années 5 .

Notes

1. Philippe Lanthier, assistant de recherche au CATIFQ, a participé à la rédaction de ce texte.

2. Appelées collocations sur la fiche en annexe; exemples: Africain francophone, cadre francophone, etc. (voir francophone, sens 2.a.).

3. Dans la citation des articles du Nouveau Petit Robert, seul le texte est fidèlement reproduit.

4. «Le français standard d'ici est la variété de français socialement valorisée que la majorité des Québécois francophones tendent à utiliser dans les situations de communication formelle.» (Conseil de la langue française $1990: 31$ )

5. À côté de ce premier modèle de fiche, nous en avons établi un second pour le vocabulaire général, de moyenne fréquence, rattaché aux divers domaines de la langue.

\section{RÉFÉRENCES}

Conseil de la langue française (1990): L'aménagement de la langue : pour une description du français québécois. Rapport et avis, $65 \mathrm{p}$.

Dictionnaire québécois d'aujourd' hui (1992) : nouvelle édition 1993, Montréal, DicoRobert.

MARTEL, Pierre et Michel THÉORET (1991): «Les bases de données textuelles et linguistiques à Sherbrooke: une banque en développement», Revue de linguistique québécoise, Montréal, UQAM, Vol. $20, \mathrm{n}^{\circ} 2$, pp. 123-143.

MARTEL, Pierre et Michel THÉORET (1993): «Modèle de description lexicographique à des fins prédictionnairiques», communication présentée au congrès de l'ACFAS, Rimouski, $16 \mathrm{p}$. 
MARTEL, Pierre (1993) : «La question de la documentation, notamment non littéraire, dans l'élaboration de textes dictionnairiques entièrement québécois», Actes $d u X X^{e}$ congrès international de linguistique et philologie romanes, T.1 IV, Section VI, Lexicographie / Galloromania, pp. 225-237.

QUEMADA, Bernard (1990) : «La nouvelle lexicographie», La Linguistica applicada, Université de Barcelone, $n^{\circ}$ 9, pp. 55-78.

\section{ANNEXE}

Eechevocable

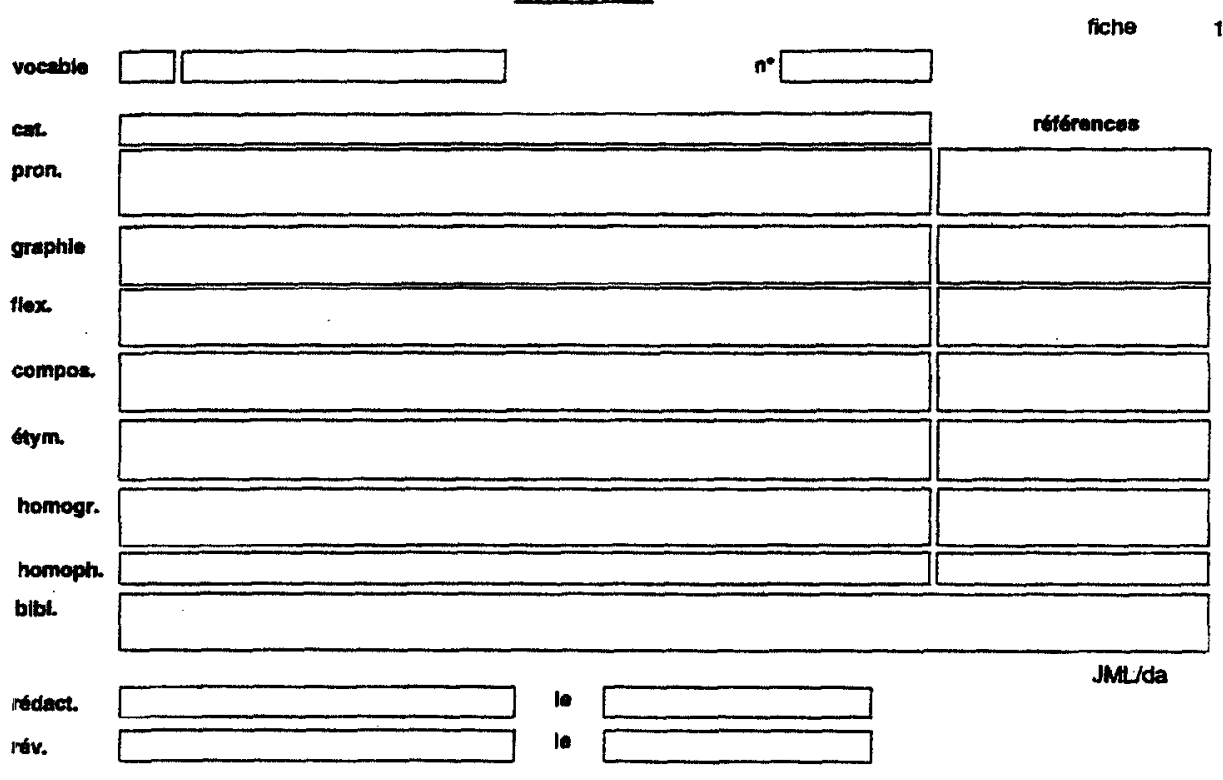


Echerecention

PREDICTIONNAIRE (CATIFQ)

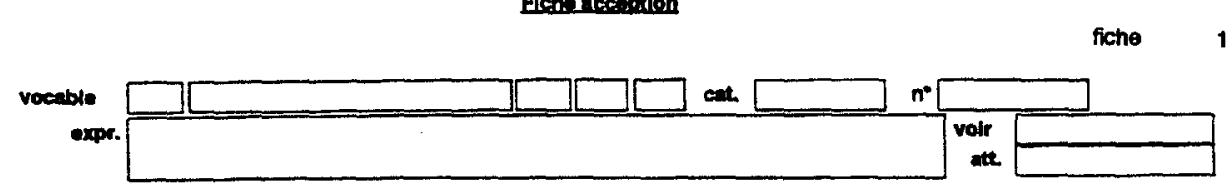

deceription

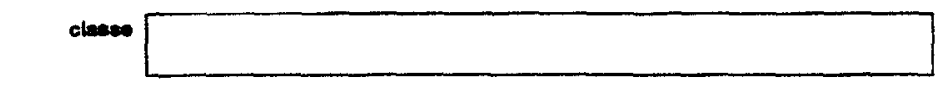

nutirences

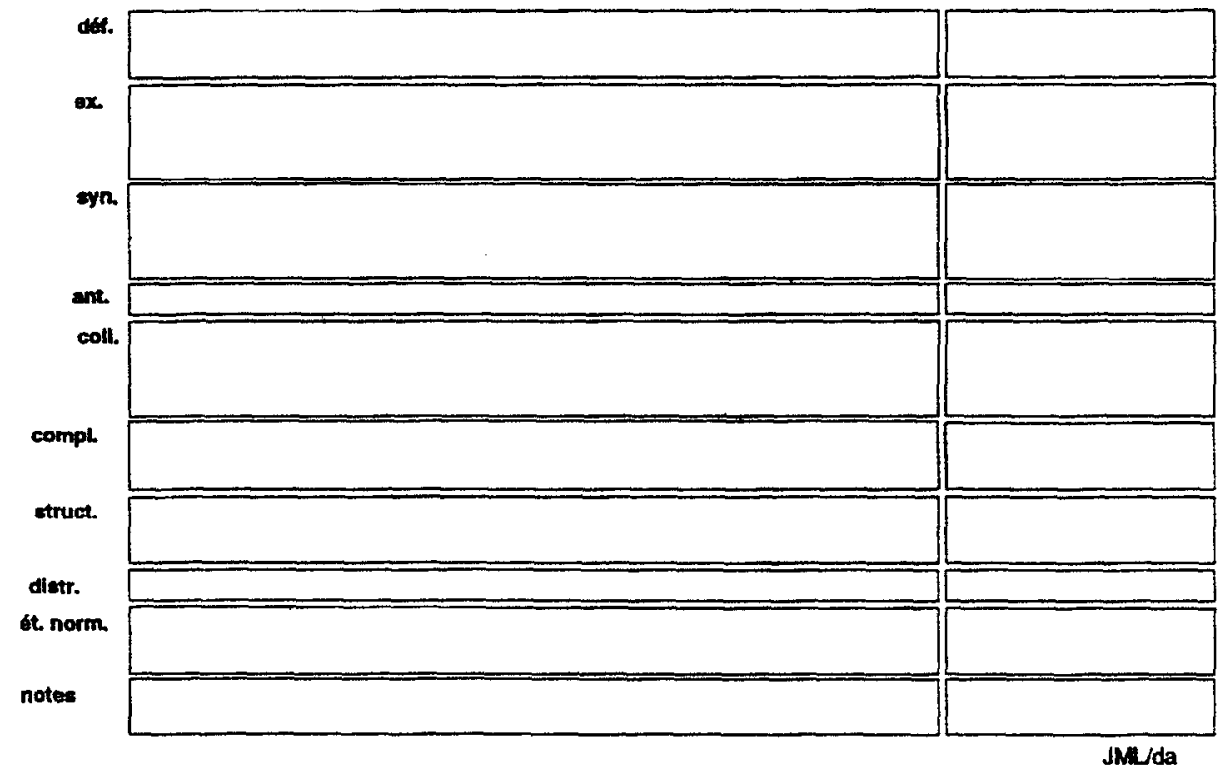

rédact.

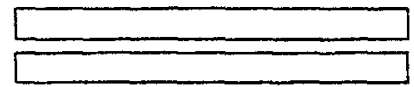

1.

rév.

le

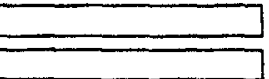

